\title{
Triagem neonatal para infecção chagásica congênita: aplicação de análise de classe latente para avaliação dos testes diagnósticos
}

\author{
Neonatal screening for congenital Chagas infection: \\ application of latent class analysis for diagnostic test evaluation
}

André Queiroz de Andrade ${ }^{1}$ e Eliane Dias Gontijo ${ }^{2}$

\begin{abstract}
RESUMO
0 presente estudo tem como objetivo avaliar os testes sorológicos convencionais usados na triagem neonatal para doença de Chagas, discutindo métodos estatísticos disponíveis. Estudou-se uma amostra aleatória dentre 23.308 recém-nascidos triados para doença de Chagas congênita por meio de três testes: imunoensaioenzimático, imunofluorescência indireta e hemoaglutinação indireta. Os dados obtidos foram analisados por diferentes metodologias estatísticas: a análise de classe latente, o teste Kappa e a análise de sensibilidade relativa. Utilizando a análise de classe latente, a maior sensibilidade foi do imunoensaioenzimático (48,6\%), seguido pela imunofluorescência indireta (39,8\%) e pela hemoaglutinação indireta (23,2\%). 0 valor Kappa foi 0,496. A razão entre as sensibilidades dos testes imunoensaioenzimático e imunofluorescência indireta foi de 92\% [0,74;1,13]. A análise de classe latente não se mostrou adequada para determinação de sensibilidade e especificidade, mas forneceu dados importantes sobre a equivalência dos testes, corroborados pela análise de sensibilidade relativa. Os resultados mostraram que o teste imunoensaioenzimático em sangueseco pode ser utilizado com a mesma segurança do teste imunofluorescência indireta.
\end{abstract}

Palavras-chaves: Doença de Chagas congênita. Análise de classe latente. Triagem neonatal. Qualidade. Técnicas e procedimentos de laboratório.

\begin{abstract}
The present study had the aim of evaluating conventional serum tests that are used in neonatal screening for Chagas disease, with a discussion on the statistical methods available. A random sample among 23,308 newborns who were screened for congenital Chagas disease was studied using the following three tests: enzyme immunoassay, indirect immunofluorescence and indirect hemagglutination. The data were analyzed by different statistical methodologies: latent class analysis, Kappa test and relative sensitivity analysis. Using latent class analysis, enzyme immunoassay had the highest sensitivity (48.6\%), followed by indirect immunofluorescence (39.8\%) and indirect hemagglutination (23.2\%). The kappa value was 0.496 . The ratio between the sensitivities of enzyme immunoassays and indirect immunofluorescence tests was $92 \%$ [0.74;1.13]. Latent class analysis was not found to be adequate for sensitivity and specificity determination, but it provided important data about the equivalence of the tests, corroborated by relative sensitivity analysis. The results showed that enzyme immunoassaying of dry blood samples can be used as safely as the indirect immunofluorescence test.
\end{abstract}

Key-words: Congenital Chagas disease. Latent class analysis. Neonatal screening. Quality. Laboratory techniques and procedures.

A doença de Chagas persiste como um problema de saúde pública prioritário na América Latina. No Brasil, seu custo médico-social é elevado, estimando-se o número de infectados em 2,5 milhões ${ }^{8}$. A partir de 1975, o controle vetorial e a melhoria das condições habitacionais e sanitárias permitiram o controle da doença. Em 2005, a Organização Panamericana da Saúde/ Organização Mundial da Saúde (OPAS/OMS) declarou o Brasil livre da transmissão pelo principal vetor da doença ${ }^{25}$.

Com a efetividade crescente no controle da transmissão transfusional nos serviços de hemoterapia, por uma rigorosa triagem soro-epidemiológica ${ }^{9}$, mecanismos alternativos de transmissã $0^{24}$ vêm sendo colocados em evidência, com destaque para a transmissão congênita, que parece ser uma das principais formas de transmissão nos dias atuais.

A taxa de transmissão vertical do Trypanosoma cruzi é baixa (em torno de $1 \%^{625}$ ). No entanto, a maioria das crianças infectadas $(60-90 \%)^{18}$ não apresenta manifestações clínicas e 0 tratamento etiológico resulta em elevados índices de cura ${ }^{3}$. Impõe-se, portanto, a escolha de estratégias de abordagem

1. Médico e Mestre em Ciência da Informação, Universidade Federal de Minas Gerais, Belo Horizonte, MG. 2. Professora associada da Faculdade de Medicina da Universidade Federal de Minas Gerais e pesquisadora do Núcleo de Ações e Pesquisa em Apoio Diagnóstico, Belo Horizonte, MG.

Apoio financeiro: Esse projeto foi financiado pelo CGDEP/ SVS/ Ministério da Saúde.

Endereço para correspondência: Dr. André Queiroz de Andrade. Rua Rogério Fajardo 180/401, Bairro Anchieta, $30310-450$ Belo Horizonte, MG.

Tel: 5531 3285-1491/55 31 9731-9753

e-mail: andrade.andreq@gmail.com

Recebido para publicação em 17/03/2008

Aceito em 09/10/2008 
que possibilitem o diagnóstico precoce e o início imediato da terapêutica (impossibilitado na gravidez ${ }^{2}$ ).

A triagem neonatal é uma estratégia de saúde pública altamente efetiva e utilizada no mundo inteiro ${ }^{26}$ para o diagnóstico de doenças tratáveis quando detectadas precocemente, reduzindo os custos e melhorando a qualidade de vida dos pacientes. O emprego de técnica sorológica de mais alto desempenho possibilita um fluxo de realização e repetição de exames mais eficiente para identificar a criança infectada. Nesse contexto, a validação permanente do teste diagnóstico utilizado é muito importante para manutenção da qualidade do serviço. No mais, possibilita a análise da viabilidade da triagem considerando a baixa prevalência da doença, o custo de cada exame e a proporção de falso-negativos e falso-positivos.

A revisão da literatura evidencia uma escassez de dados sobre avaliação de concordância entre testes sorológicos em sangue seco para o diagnóstico da infecção chagásica. 0 presente estudo faz parte de uma avaliação maior sobre a transmissão congênita da doença de Chagas em Minas Gerais* e tem como objetivo avaliar os testes diagnósticos usados na triagem neonatal, discutindo métodos estatísticos disponíveis para assegurar a qualidade do diagnóstico.

\section{MATERIAL E MÉTODOS}

Desenho do estudo. Estudo transversal, pela metodologia da triagem neonatal, de crianças expostas à infecção chagásica em Minas Gerais, utilizando três testes diagnósticos em sangue seco: imunofluorescência indireta (IFI), imunoensaioenzimático (ELISA), hemaglutinação indireta (HAI). Para avaliação dos exames utilizaram-se os testes estatísticos: Kappa, análise de classe latente e comparação da sensibilidade relativa de cada um dos testes.

Testes diagnósticos. Os testes em sangue-seco para triagem de doença de Chagas congênita (DCC) são baseados na pesquisa de IgG anti-Trypanosoma cruzi. Uma vez que tal molécula tem livre passagem pela placenta materna, considerouse a identificação de um teste positivo no recém-nascido como sugestivo de infecção materna.

As amostras coletadas foram submetidas primeiramente ao ELISA, em laboratório próprio do Núcleo de Ações e Pesquisa em Apoio Diagnóstico (NUPAD)/FM/UFMG, utilizando-se o kit comercial Chagatek ELISA (Buenos Aires, Argentina). As amostras com títulos superiores a 0,6 foram consideradas positivas. Todas as amostras com resultado alterado no teste ELISA e $20 \%$ das amostras negativas foram submetidas aos testes de IFI e HAI no laboratório de sorologia de doença de Chagas da Fundação Ezequiel Dias (FUNED), Minas Gerais. 0 teste de IFI foi usado em diversos estudos de triagem neonatal ${ }^{5}$ e tem seu uso consagrado. 0 teste de hemaglutinação foi realizado de acordo com a técnica de

*Gontijo ED, Andrade GM, Santos SE, Galvão LMC, Moreira EF. Triagem neonatal para infecção chagásica em Minas Gerais: transmissão congênita e mapeamento das áreas endêmicas. Revista de Epidemiologia e Serviços de Saúde ( aguardando parecer)
Kienerim-Saavedra ${ }^{13}$, utilizando-se 0 kit comercial da Biomérieux Brasil S/A (Jacarepaguá, RJ). Os resultados das amostras com titulação igual a ou maior do que 1:16 foram considerados positivos.

Todas as mães cujos filhos tiveram pelo menos um teste alterado foram submetidas ao exame em soro (Figura 1) para confirmação da infecção materna. Foram realizados nestas amostras os testes ELISA, IFI e HAI, considerados como teste referência neste estudo.

Para definição de infecção chagásica utilizou-se o critério de caso da $\mathrm{OMS}^{29}$, que exige a positividade em pelo menos dois testes de técnicas diferentes. 0 presente estudo compara, portanto, 0 resultado em sangue-seco do neonato (que sugere a infecção materna) e a confirmação do diagnóstico da mãe, pela sorologia, que identifica a criança exposta.

Amostra. Estudou-se uma amostra aleatória dentre 23.308 recém-nascidos (preconiza-se a coleta do sangue no $5^{\circ}$ dia de vida) participantes do Programa Estadual de Triagem Neonatal em Minas Gerais (PETN-MG), no período de 8 de agosto a 9 de setembro de 2005. As amostras de sangue de calcanhar dos RN foram enviadas ao NUPAD, órgão da Faculdade de Medicina da Universidade Federal de Minas Gerais, referência estadual do Ministério da Saúde em triagem neonatal.

Para a análise foram selecionadas todas as amostras negativas no teste ELISA submetidas aos outros dois testes ( $20 \%$ das amostras negativas, 4.658 amostras) e foi aleatorizada a mesma proporção de testes alterados no ELISA (20\% de 269, ou 50 amostras) (Figura 1). Realizou-se esse procedimento com o objetivo de simular uma amostragem aleatória da população triada.

Análise estatística. Os dados obtidos foram analisados por diferentes testes estatísticos visando avaliar a aplicabilidade desses testes no presente desenho de estudo. Para a validação dos testes, a sensibilidade e especificidade de cada teste foram estimadas utilizando-se o método de análise de classe latente (ACL). A análise de classe latente é uma modelagem matemática baseada na suposição que uma variável não-observada (latente) determina as associações entre as variáveis observadas ${ }^{22}$. A adequação deste modelo exige que as variáveis analisadas sejam independentes entre si (suposição de independência condicional) ${ }^{27}$. Toft e $\operatorname{cols}^{27}$ definem que independência condicional entre dois testes significa que, quando é sabido o status da doença de um individuo testado, a probabilidade do resultado de um teste não é afetado pelo conbecimento do resultado de outro.

Para a verificação desta suposição foi utilizada a comparação entre frequiências marginais das tabelas $2 \mathrm{X} 2$ observadas e as tabelas preditas pelo modelo para cada par de variáveis manifestas ${ }^{23}$. Comparou-se a estimativa da ACL com a informação disponível na literatura sobre os referidos testes.

A segunda parte da análise consistiu na comparação entre os testes. Realizou-se o teste Kappa para avaliar a concordância entre os testes ELISA e IFI (devido à maior importância destes testes na prática da triagem neonatal). Comparou-se a sensibilidade relativa de cada um dos testes, definido por Cheng e Macaluso ${ }^{7}$. Esta metodologia considera a informação dada pelo teste referência 


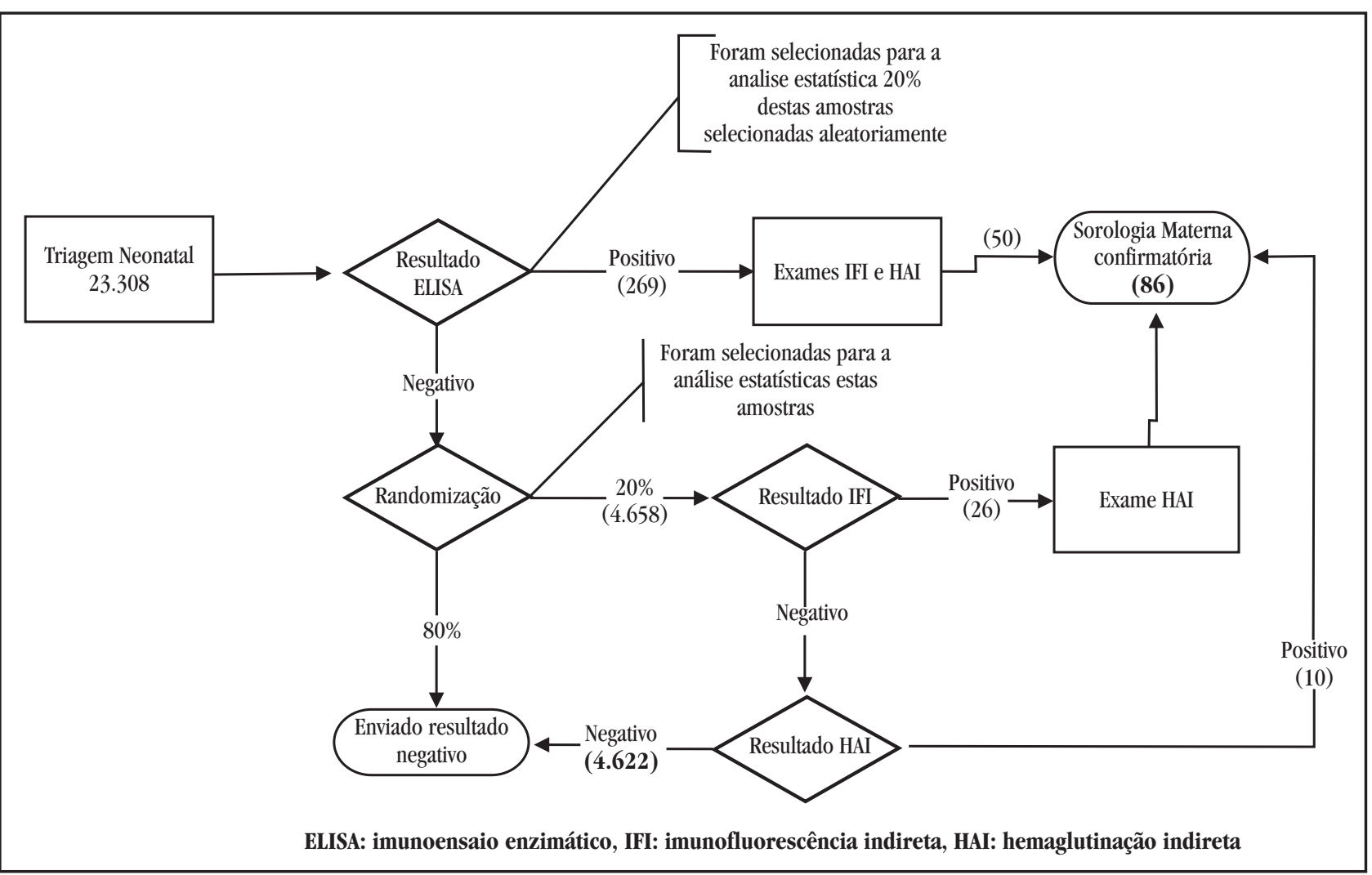

Figura 1 - Fluxograma das amostras de crianças suspeitas de infecção chagásica, identificadas pela triagem neonatal em Minas Gerais.

e foi escolhida devido ao desenho do estudo (confirmação por teste-referência apenas dos triados positivos).

Para análise estatística utilizaram-se softwares SPSS (software proprietário), LEM (disponível no site http://www.uvt.nl/faculteiten/ fsw/organisatie/departementen/mto/software2.html) para a análise de classe latente e o Condep (disponível no site http://ourworld. compuserve.com/homepages/jsuebersax/condep.zip) para o teste de independência condicional.

Aspectos éticos. Este estudo integra o projeto Transmissão congênita da infecção chagásica em Minas Gerais que foi aprovado pelo COEP - Comitê de Ética em Pesquisa da UFMG com o número de protocolo: ETIC 260/03.

\section{RESULTADOS}

Foram testadas 4.708 amostras de sangue seco analisadas nos três testes (ELISA, IFI e HAI) e os resultados encontram-se na Tabela 1. Dessas amostras, 86 tiveram pelo menos um resultado alterado e foram encaminhadas para o teste sorológico. Das 86 positivas, foram recebidas 79 amostras em soro, sendo 27 (34\%) positivas e $52(66 \%)$ negativas.

Para a análise dos testes, consideraram-se para efeito da análise os resultados indeterminados como negativos. A escolha desse critério se deu devido às recomendações da OMS que consideram apenas os resultados positivos para o diagnóstico.

Análise de classe latente. Para analisar a sensibilidade e especificidade de cada teste, aplicou-se o teste estatístico ACL.
Tabela 1 - Resultado dos testes utilizados para detecção de IgG antiTrypanosoma cruzi nas amostras de sangue seco de 4.708 recém-nascidos participantes do Programa de Triagem Neonatal em Minas Gerais no período de 8 de agosto a 9 de setembro de 2005.

\begin{tabular}{|c|c|c|c|c|c|c|}
\hline \multirow{3}{*}{$\begin{array}{l}\text { Testes para detecção de IgG } \\
\text { anti-Trypanosoma cruzi } \\
\text { (sangue seco) }\end{array}$} & \multicolumn{6}{|c|}{ Resultados } \\
\hline & \multicolumn{2}{|c|}{ positivo } & \multicolumn{2}{|c|}{ indeterminado } & \multicolumn{2}{|c|}{ negativo } \\
\hline & $\mathrm{n}^{0}$ & $\%$ & $\mathrm{n}^{0}$ & $\%$ & $\mathrm{n}^{0}$ & $\%$ \\
\hline Imunoensaio enzimático & 44 & 0,9 & 6 & 0,1 & 4.658 & 98,9 \\
\hline Imunofluorescência indireta & 36 & 0,8 & 11 & 0,2 & 4.661 & 99,0 \\
\hline Hemaglutinação indireta & 21 & 0,4 & 1 & 0,0 & 4.686 & 99,5 \\
\hline
\end{tabular}

A Tabela 2 apresenta as frequiências observadas e estimadas no modelo de análise de classe latente (supondo que todos os resultados observados são independentes entre si). Para testar a dependência condicional, a matriz de correlação dos resíduos foi analisada numericamente e graficamente. Não se detectou

Tabela 2 - Frequiências observadas e estimadas pelo modelo de classe latente e o resíduo (diferença entre os valores observados e estimados) padrão.

\begin{tabular}{lcrrrc}
\hline ELISA & IFI & HAI & Observado & Estimado & Resíduo padrão \\
\hline 1 & 1 & 1 & 12.000 & 4.059 & 3.942 \\
1 & 1 & 2 & 8.000 & 13.439 & -1.484 \\
1 & 2 & 1 & 0.000 & 6.148 & -2.479 \\
1 & 2 & 2 & 24.000 & 20.354 & 0.808 \\
2 & 1 & 1 & 0.000 & 4.292 & -2.072 \\
2 & 1 & 2 & 16.000 & 14.210 & 0.475 \\
2 & 2 & 1 & 9.000 & 6.501 & 0.980 \\
2 & 2 & 2 & 4.639 .000 & 4.638 .997 & 0.000 \\
\hline
\end{tabular}

ELISA: imunoensaio enzimático, IFI: imunofluorescência indireta, HAI: hemaglutinação indireta. 
dependência significante entre as variáveis, apesar de o escore z sugerir dependência condicional entre os testes 2 (IFI) e 3 (HAI). A Tabela 3 e a Figura 2 exploram a análise da independência entre as variáveis.

Tabela 3 - Teste de independência condicional, utilizando abordagem de comparação entre valores residuais observados (reais) e os preditos pelo modelo de classe latente para cada par de variável manifesta programa Condep.

\begin{tabular}{lcccccc}
\hline \multicolumn{2}{l}{ Variáveis } & $\begin{array}{c}\text { Log odds ratio } \\
\text { esperado }\end{array}$ & $\begin{array}{c}\text { Erro } \\
\text { padrão }\end{array}$ & $\begin{array}{c}\text { Log odds ratio } \\
\text { observado }\end{array}$ & Escore z & Valor p \\
\hline ELISA & IFI & 5,11 & 0,386 & 5,49 & 0,98 & 0,3271 \\
ELISA & HAI & 4,87 & 0,470 & 5,27 & 0,85 & 0,3953 \\
IFI & HAI & 4,71 & 0,485 & 5,56 & 1,74 & 0,0819 \\
\hline
\end{tabular}

ELISA: imunoensaio enzimático, IFI: imunofluorescência indireta, HAI: hemaglutinação indireta

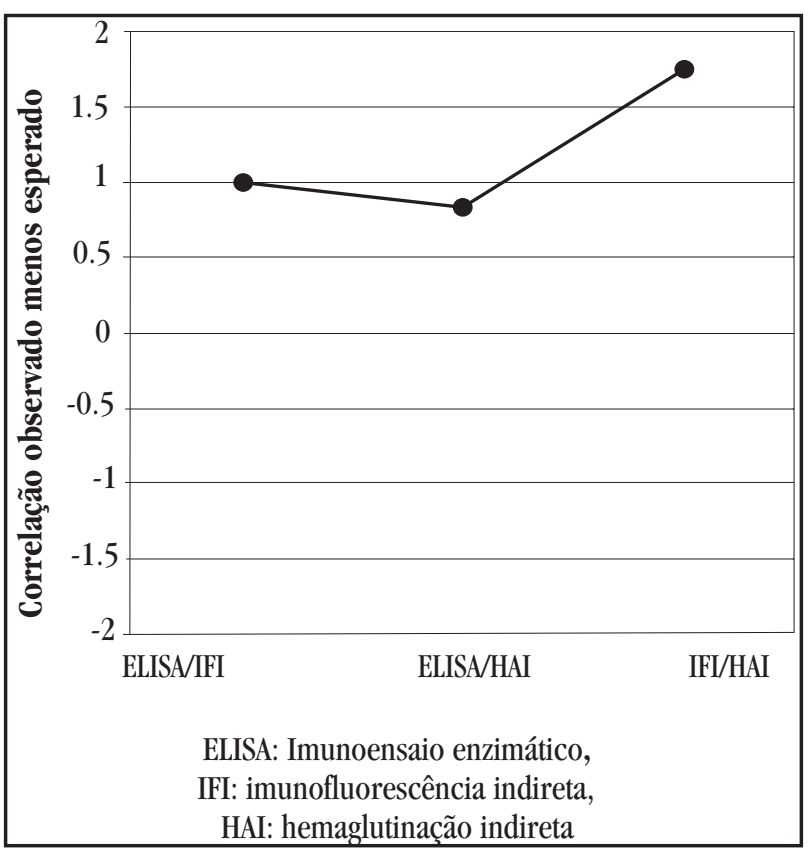

Figura 2 - Correlação dos resíduos entre os testes utilizados na triagem neonatal da doença de Chagas congênita, utilizando o modelo de classe latente, no qual se observa que quanto maior a distância dos pontos em relação ao valor 0 , maior a probabilidade de dependência entre os testes.

A prevalência da doença de Chagas em gestantes encontrada pela análise foi de 1,9\%. A maior sensibilidade foi do ELISA (48,6\%), seguido pelo IFI (39,8\%) e pelo HAI (23,2\%) (Tabela 4).

Comparação entre os testes - sensibilidade proporcional. Na segunda parte da análise, os testes ELISA (principal teste utilizado na triagem por sua capacidade de automatização) e o IFI (teste com maior experiência na literatura, seja em sorologia ou sangue-seco) foram posteriormente comparados entre si. Os resultados em sorologia foram considerados como referência - padrão-ouro.

A razão entre as sensibilidades dos testes ELISA e IFI foi de 92\% - IC 95\% [0,74; 1.13]. A razão entre os resultados falsopositivos dos testes ELISA e IFI foi de 190\% - IC 95\% [0,88; 4,09]. A Tabela 5 mostra a referência utilizada. Realizou-se ainda o teste Kappa para avaliar a concordância entre estes testes, tendo sido obtido o valor de $\mathbf{0 , 4 9 6}$, considerado concordância mediana.
Tabela 4 - Análise da sensibilidade e especificidade, pela análise de classe latente, dos testes utilizados na identificação de IgG antiTrypanosoma cruzi nas amostras de sangue seco de 4.708 recém-nascidos participantes do Programa de Triagem Neonatal em Minas Gerais no período de 8 de agosto a 9 de setembro de 2005.

Testes para detecção de IgG anti-Trypanosoma cruzi Sensibilidade Especificidade

\begin{tabular}{lll} 
& $(\%)$ & $(\%)$ \\
\hline ELISA & 48,6 & 100 \\
IFI & 39,8 & 100 \\
HAI & 23,2 & 100
\end{tabular}

ELISA: imunoensaio enzimático, IFI: imunofluorescência indireta, HAI: hemaglutinação indireta

Tabela 5 - Freqüiencias marginais dos resultados do teste de imunoensaio enzimático e imunofluorescência indireta anti-Trypanosoma cruzi em sangue seco de pacientes com exames confirmatórios em soro*.

\begin{tabular}{|c|c|c|c|c|c|c|c|}
\hline & \multicolumn{7}{|c|}{ Sorologia confirmatória } \\
\hline & \multicolumn{3}{|c|}{ infecção chagásica positiva } & & \multicolumn{3}{|c|}{ infecção chagásica negativa } \\
\hline & IFI + & IFI - & total & & $\mathrm{IFI}+$ & IFI - & total \\
\hline ELISA + & 20 & 2 & 22 & ELISA + & 0 & 19 & 19 \\
\hline ELISA - & 4 & $?$ & & ELISA - & 10 & $?$ & \\
\hline Total & 24 & & & Total & 10 & & \\
\hline
\end{tabular}

* Uma paciente com sorologia positiva foi excluída desta análise pois foi negativa em ambos os testes (ELISA e IFI)

ELISA: imunoensaio enzimático, IFI: imunofluorescência indireta.

\section{DISCUSSÃO}

A triagem neonatal oferece possibilidade real de cura definitiva para a doença de Chagas congênita, mediante adequado tratamento específico das crianças com infecção confirmada. Para o ótimo funcionamento de um sistema de triagem, é necessário que o laboratório esteja plenamente capacitado para realização do exame, seguindo as recomendações de capacitação de pessoal, controle de qualidade e realização periódica de testes de proficiência. 0 controle de qualidade de tal laboratório deve incluir, além da análise de amostras-controle e adoção de padrões, consideração constante da sensibilidade, especificidade, valor preditivo positivo (VPP) e negativo (VPN) ${ }^{20}$.

A determinação de tais parâmetros está longe de ser trivial. A utilização de parâmetros universais de sensibilidade e especificidade pode não se mostrar a mais adequada pois, além de particularidades na coleta e análise do teste, como habilidade do coletor e temperatura de conservação da amostra, outros aspectos podem levar a variações dos parâmetros como características demográficas e a gravidade da doença na amostra estudada ${ }^{28}$. Isto significa que, apesar de, conceitualmente, sensibilidade e especificidade serem características estáveis do teste, os valores determinados em um laboratório podem não ser válidos para outros.

Dado a raridade das doenças detectadas pela triagem neonatal, uma grande preocupação é o tamanho de amostra requerido para estudos de validação de testes diagnósticos. Algumas abordagens alternativas visam diminuir o tamanho da amostra ${ }^{19}$, embora necessitem de grande quantidade de testes confirmatórios (muitas vezes impossibilitados por custo e realidade regional). Para contornar essas dificuldades, uma solução seria a análise das 
amostras sem o teste referência (padrão-ouro). Este assunto vem sendo crescentemente estudado devido à percepção de falibilidade dos testes referência, além de questões éticas na realização de estudos com seres humanos. Dentre as abordagens sugeridas na literatura, duas foram estudadas: a análise de classe latente e o teste Kappa de concordância.

A análise de classe latente é uma técnica matemática bastante utilizada, com várias publicações na área de epidemiologia ${ }^{4} 1522$. A análise de classe latente permite a estimativa da sensibilidade e especificidade de cada um dos testes, e a prevalência da doença na população estudada. No entanto, para que essa abordagem seja fidedigna, tornam-se necessárias algumas suposições, conhecidas como o paradigma de Hui e Walter ${ }^{12}$. Dentre essas suposições estão a presença de um número mínimo de variáveis ou populações observadas (ex. 3 testes e 1 população, ou 2 testes e 2 populações) e a suposição de independência condicional.

No presente estudo, foram observadas algumas limitações para o uso da análise de classe latente na triagem neonatal. A coleta dos programas de triagem neonatal é feita em sangue-seco; portanto, a análise é feita sobre a mesma amostra biológica, ferindo conceitualmente a hipótese de independência condicional. Outro problema reside no fato de os testes utilizados pesquisarem a mesma variável biológica (presença de IgG anti-Trypanosoma cruzi), agravante também verificado em outros artigos ${ }^{1522}$.

0 método gráfico para visualização do modelo ${ }^{23}$ (Figura 2) mostra associação entre os testes IFI e HAI, introduzindo, possivelmente, uma violação do pressuposto de independência condicional. 0 número de variáveis avaliadas nesse estudo não possibilitou ajuste do modelo, o que possivelmente explica resultados inesperados de sensibilidade e especificidade dos testes diagnósticos. Além das limitações apresentadas, a reduzida frequiência de eventos observada em algumas caselas constitui restrição para análises que utilizam a distribuição qui-quadrado.

A experiência de vários autores e as limitações observadas justificam análise cautelosa dos resultados ${ }^{11}$. Pepe e cols ${ }^{21}$ argumentam que mesmo se todas as suposições fossem atingidas, a análise de classe latente ainda poderia fornecer dados incorretos sobre a acurácia do exame. Esses autores demonstram que pequenas alterações na correlação entre os testes podem causar grandes alterações na estimativa de indivíduos verdadeiramente positivos.

Como as características do presente estudo são comuns a todas as doenças da triagem neonatal (baixa prevalência, dificuldade da coleta em soro, realização de vários exames em uma mesma amostra biológica utilizando a mesma variável biológica), considera-se que a análise de classe latente não é a ferramenta mais adequada para este tipo de análise de acurácia de teste diagnóstico. Bertrand e cols ${ }^{1}$ argumentam que a ACL pode ser mais útil na análise de concordância entre observadores do que na avaliação de análise de acurácia. A análise de classe latente mostra maior sensibilidade do ELISA em relação ao IFI (teste já consagrado em papel-filtro), ao passo que a análise do Kappa mostra concordância mediana entre os dois testes $(\kappa=0.496)$. A análise de classe latente tem a vantagem de permitir a análise da concordância em determinados parâmetros de interesse (sensibilidade, especificidade) facilitando a tomada de decisão consciente.
Existem alternativas para driblar a dificuldade de avaliação da acurácia de um teste, mantendo-se a confiabilidade da triagem. A introdução de um segundo teste de triagem, já validado anteriormente ou utilizado habitualmente em serviços de referência, pode fornecer dados suficientes. Como, na triagem, a sensibilidade do teste é o fator mais importante ${ }^{14}$, um segundo teste deve, pelo menos, ter sensibilidade semelhante à do teste utilizado. Essa abordagem não determina a sensibilidade e especificidade, mas a comparação entre os dois testes e a sensibilidade e especificidade proporcionais.

No caso da doença de Chagas, a amostragem em duas fases com a confirmação diagnóstica somente das amostras com resultado positivo foi o método escolhido. Cheng e Macaluso propõem uma forma de comparar os testes quando o padrãoouro é realizado somente nos resultados positivos ${ }^{7}$. Essa forma de análise é bastante direta e fornece as informações necessárias para a decisão entre dois testes. A redução de custo é substancial e não há perda na qualidade da decisão.

Os resultados apresentados na ACL são conflitantes com os achados da literatura ${ }^{1617}$. A razão entre as sensibilidades mostra boa concordância entre a sensibilidade e proporção de falsopositivos dos testes IFI e ELISA. Este resultado é suficiente para a adoção segura do teste de ELISA na triagem, que se mostrou equivalente ao IFI.

A alocação criteriosa de recursos é de suma importância na sustentabilidade de qualquer serviço de saúde. A triagem neonatal não é exceção e devem ser observados criteriosamente os investimentos no sentido de buscar a máxima qualidade com o mínimo de recursos. 0 presente estudo sugere que uma metodologia simples para comparação entre testes é suficiente para validar o uso de um exame diagnóstico. 0 aumento da complexidade da análise estatística deve ser feito somente nos casos em que avaliações mais simples não são esclarecedoras. Procedimentos estatísticos complexos podem induzir viés na análise (caso suas limitações não sejam consideradas) além de necessitarem de profissionais altamente especializados não disponíveis em todos os serviços.

Concluindo, a análise de classe latente não se mostrou adequada para determinação de sensibilidade e especificidade, mas forneceu dados importantes sobre a equivalência dos testes. A razão entre as sensibilidades e proporção de falso-positivos corroborou essa equivalência. Os resultados mostraram que o teste ELISA em sangue-seco pode ser utilizado com a mesma segurança do teste IFI. No presente estudo, o ELISA apresentou número maior de testes falso-positivos, o que é esperado de testes de alta sensibilidade, utilizados em triagem.

\section{REFERÊNCIAS}

1. Bertrand P, Benichou J, Grenier P, Chastang C. Hui and Walter's latent-class reference-free approach may be more useful in assessing agreement than diagnostic performance. Journal of Clinical Epidemiology 58:688-700, 2005.

2. Blanco SB, Segura EL, Cura EN, Chuit R, Tulián L, Flores I, Garbarino G, Villalonga JF, Gürtler RE. Congenital transmission of Trypanosoma cruzi: an operational outline for detecting and treating infected infants in north-western Argentina. Tropical Medicine and International Health 5:293-301, 2000.

3. Blanco SB, Segura EL, Gurtler RE. Control of congenital transmission of Trypanosoma cruzi in Argentina. Medicina (reumenes) 59:138-142, 1999. 
4. Boelaert M, Aoun K, Liinev J, Goetghebeur E, Van der Stuyft P. The potential of Latent Class Analysis in diagnostic test validation for canine Leishmania infantum infection. Epidemiology and Infection 123:499-506, 1999

5. Camargo Neto E, Rubin R, Schulte J, Giugliani R. Newborn screening for congenital infectious diseases. Emerging Infectious Diseases 10:1068-1073, 2004.

6. Carlier Y, Torrico F. Congenital infection with Trypanosoma cruzi: from mechanisms of transmission to strategies for diagnosis and control. Revista da Sociedade Brasileira de Medicina Tropical 36:767-771, 2003.

7. Cheng H, Macaluso M. Comparison of the Accuracy of Two Tests with a Confirmatory Procedure Limited to Positive Results. Epidemiology 8:104-106, 1997.

8. Dias JCP. 0 Controle da Doença de Chagas no Brasil. In: Silveira AC, (ed) 0 Controle da Doença de Chagas nos Países do Cone Sul da América, Faculdade de Medicina do Triângulo Mineiro, Uberaba, p.146-250, 2002.

9. Dias JCP, Schofield CJ. Controle da transmissão transfusional da Doença de Chagas na iniciativa do Cone Sul. Revista da Sociedade Brasileira de Medicina Tropical 31:373-383, 1998 .

10. Freiliji H, Altchehj J, Corral R. Respuesta al tratamento em niños com infección chagásica congenita em zona no endémica. Medicina (reumenes) 50:389, 1990.

11. Goetghebeur E, Liinev J, Boelaert M, Van der Stuyft P. Diagnostic test analyses in search of their gold standard: latent class analyses with random effects. Statistical Methods in Medical Reserch 9:231-248, 2000.

12. Hui SL, Walter SD. Estimating the Error Rates of Diagnostic-Tests. Biometrics 36:167-171, 1980.

13. Knierim F, Saavedra P. Tecnica de la reaccíon de hemaglutinación aplicada al diagnóstico serológico de las parasitoses. Boletín Chileno de Parasitología 21:39-44, 1965.

14. Kwon C, Farrel PM. The magnitude and challenge of false-positive newborn screening test results. Archives of Pediatrics \& Adolescent Medicine $154: 714-718,2000$

15. Langhi Junior DM, Bordin JO, Castelo A, Walter SD, Moraes-Souza H, Stump RJ. The Application of Latent Class Analysis for Diagnostic Test Validation of Chronic Trypanosoma cruzi Infection in Blood Donors. The Brazilian Journal of Infectious Diseases 6:181-187, 2002.

16. Luquetti AO, Ponce C, Ponce E, Esfandiari J, Schijman A, Revollo S, Anez N, Zingales B, Ramgel-Aldao R, Gonzalez A, Levin MJ, Umezawa ES, Silveira JF. Chagas' disease diagnosis: a multicentric evaluation of Chagas Stat-Pak, a rapid immunochromatographic assay with recombinant proteins of Trypanosoma cruzi. Diagnostic Microbiology Infectious Disease 46:265-271, 2003.

17. Machado-Coelho GL, Vitor RW, Chiari Cde A, Antunes CM. Validity of serology for American trypanosomiasis with eluates from filter paper. Memórias do Instituto Oswaldo Cruz 90:59-64, 1995.

18. Moya PR. Enfermedad de Chagas congénita. Relatório final da XVI Reunião Anual de Pesquisa Aplicada em Doença de Chagas e leishmanioses, Uberaba, p. 3, 2000.

19. Obuchowski NA, Zhou XH. Prospective studies of diagnostic test accuracy when disease prevalence is low. Biostatistics 3:477-492, 2002.

20. Pass KA, Lane PA, Fernhoff PM, Hinton CF, Panny SR, Parks JS, Pelias MZ, Rhead WJ, Ross SI, Wethers DL, Elsas LJ. US Newborn Screening System Guidelines II: Follow-up of children, diagnosis, management, and evaluation - Statement of the Council of Regional Networks for Genetic Services (CORN). Journal of Pediatrics 137:S1-\$46, 2000.

21. Pepe MS, Alonzo TA. Comparing disease screening tests when true disease status is ascertained only for screen positives. Biostatistics 2:249-260, 2001.

22. Pirard M, Iihoshi N, Boelaert M, Basanta P, Lopez F, Van der Stuyft P. The validity of serologic tests for Trypanosoma cruzi and the effectiveness of transfusional screening strategies in a hyperendemic region. Transfusion 45:554-561, 2005.

23. Qu YS, Tan M, Kutner MH. Random effects models in latent class analysis for evaluating accuracy of diagnostic tests. Biometrics 52:797-810, 1996.

24. Schmunis GA. Tripanosomíase americana: seu impacto nas Américas e perspectivas de eliminação. In: Dias JCP, Coura JR (eds) Clínica e terapêutica da doença de Chagas, Fiocruz, Rio de Janeiro, p.11-24, 1997.

25. Secretaria de Vigilância em Saúde. Consenso Brasileiro em Doença de Chagas. Revista da Sociedade Brasileira de Medicina Tropical 38 supl 3:1-29, 2005.

26. Therrell BL. Challenges and opportunities in establishing and maintaining newborn screening systems. Southeast Asian Journal of Tropical Medicine Public Health 34 supl:6-12, 2003.

27. Toft N, Jorgensen E, Hojsgaard S. Diagnosing diagnostic tests: evaluating the assumptions underlying the estimation of sensitivity and specificity in the absence of a gold standard. Preventive Veterinary Medicine 68:19-33, 2005.

28. Whiting P, Rutjes AWS, Reitsma JB, Glas AS, Bossuyt PMM, Kleijnen J. Sources of variation and bias in studies of diagnostic accuracy - A systematic review. Annals of Internal Medicine 140:189-202, 2004

29. World Health Organization Expert Committee. Control of Chagas disease. Geneva: World Health Organization 2002. 